\title{
40. COMMISSION DE RADIO-ASTRONOMIE
}

Président: M. J. L. Pawsey.

Membres: MM. Appleton, Baltá, Blackett, Bolton, Hanbury Brown, Burrows, Mme Carpenter, MM. Denisse, Greenstein, Harang, Hatanaka, Hagen, Hewish, Hey, Khaikin, Laffineur, Lemoine †, Link, Lovell, McKinley, Martyn, Millman, Mills, Mitra, Nicolet, Oort, Owren, J. R. Pierce, Rakshit, Reber, Righini, Rydbeck, Ryle, Seeger, A. H. Shapley, Shklovsky, F. G. Smith, Tuominen, van de Hulst, Williamson.

\section{INTRODUCTION}

Since its birth in I932 with Jansky's discovery of cosmic radio waves, radio astronomy has developed sufficiently to permit an assessment of its place in astronomy. It is now clear that radio observations can supply important information not available optically so that the combination of radio with optical observations is indispensable in furthering our understanding of the universe. This is now widely recognized as evidenced by the existence of numerous radio observatories throughout the world. There are at least thirty-two of these distributed among fourteen countries. But it is only recently that trained astronomers have begun to play an effective role in the science. The early discoveries were made by American radio engineers; most of the currently available observations were made by Australian and English radio physicists; we are now at the stage where experienced astronomers, especially from the U.S.S.R., the U.S.A., and Holland, are making major contributions, particularly in interpretation. Radio astronomy, if it is to develop properly, must depend on a blending of radio invention and astronomical insight.

There is already a considerable literature on radio astronomy, including recent books and integrating papers (see pp. 574-5 below). In this report we shall not attempt the detailed account of recent work, which is the proper function of these papers, but present a very broad outline in an attempt to set the subject in perspective and to pick out the main questions of today. A further section deals with international collaboration, and some bibliographical notes are appended.

\section{An Outline of Radio Astronomy (I954)}

Radio astronomy may be defined as astronomy utilizing observations of radio waves. These observations fall into two distinct categories: observations of radio waves emitted naturally by the astronomical bodies studied, and observations of radio echoes. The first, analogous to those of optical astronomy, have been applied to the Moon, the Sun, objects in our galaxy, and external galaxies. The second, those of the radar method, are inherently limited to the nearer objects and so far have been applied in astronomy only to meteors and the Moon. Thus the great bulk of observations are based on naturally emitted radio waves. The value of the observations depends on the great difference in wave-length between radio waves and light, differences which involve radical differences in emission and propagation so that radio observations yield information complementary to that obtained from light.

Matter emits radio waves thermally in accordance with the normal radiation laws. The spectrum of this radiation in astronomy is usually continuous, but a single spectral line, the $\mathrm{r} 42 \mathrm{O} \mathrm{Mc}$./sec. line of atomic hydrogen, is found in emission and absorption from interstellar matter. In addition non-thermal emission mechanisms must operate in the Sun and cosmic sources. In cosmic sources the spectrum of the non-thermal component is continuous; in the Sun it can show narrow bands, but the frequencies of such bands continually change.

Radio propagation differs from optical propagation in two important respects. First, 
highly rarefied ionized gases which freely transmit light may absorb and emit, or be completely opaque to, radio waves. Secondly, clouds of solid particles (e.g. interstellar dust clouds) which absorb light may be transparent to radio waves.

Radio observations have been made of the galaxy and external galaxies, of the Sun, of the Moon, and of meteors. In addition propagation effects in the terrestrial ionosphere have been recognized and applied to yield information about it. Brief résumés of current knowledge of each of these subjects are given below.

\section{Cosmic radio waves: the continuous spectrum}

The continuous radiation is distributed over the sky with a concentration towards the plane of the Milky Way and a dominant maximum in the direction of the galactic centre.

This distribution conforms sufficiently well with the disposition of our galaxy to leave no doubt that most of the radiation originates in matter distributed widely throughout the galaxy. It is not yet possible to say to what extent the distribution conforms with those of particular classes of objects because the resolution of the equipment used for most of the published surveys has been inadequate (beam-widths of $10^{\circ}$ or $20^{\circ}$ ). The published contours may be seriously in error. There must also be an extragalactic component, but its magnitude is uncertain.

A most unexpected feature of the radiation is its high intensity at the lower frequencies. At $9 \mathrm{Mc}$./sec., for example, the brightness temperature over the whole sky averages about a million degrees Kelvin. Theory shows that ionized interstellar hydrogen, Hir regions, must emit and absorb radio waves, but these high brightness temperatures give conclusive evidence for the existence of a further, non-thermal, emission mechanism. The nature of this mechanism is still unknown. An interesting hypothesis which links these very high brightness temperatures with the tremendous energies of cosmic rays was put forward by $\mathrm{H}$. Alfvén and $\mathrm{N}$. Herlofson ( $\mathrm{r}$ ) in relation to discrete sources. They suggested that the emission may be from electrons with relativistic energies spiralling round lines of magnetic force in interstellar space. This mechanism has been further studied and extended to other circumstances, even to clusters of galaxies, by other workers, notably by I. S. Shklovsky in a series of papers (2).

The spectra from different parts of the sky differ and it is from a study of such clues that one may hope to determine the relative roles of thermal and non-thermal emission and of absorption. Similarly polarization may give important clues but no observation of polarization which is other than random has yet been described. This may either be because the radiation is emitted with random polarization or because, in the observations, a large number of independent sources combined to give a random resultant.

Observàtions indicate numerous discrete sources of small angular extent superposed on a continuous background. But the angular resolution is not sufficient to decide if the background is really continuous or made up of numerous faint sources similar to the known ones. Positions of one or two hundred have been published and several dozen have been identified with optical objects. These numbers may be expected soon to increase greatly owing to observations taken with the large Cambridge and Sydney aerial systems. M. Ryle has stated that he expects to locate I700 from the Cambridge work. Flux densities range up to about that of the Sun. Observed angular sizes range from one minute of arc to several degrees, and this suggests that none is of stellar dimensions. The sources are distributed widely over the sky and are believed to fall into two classes (3): a more intense class concentrated towards the galactic plane and towards the centre of the galaxy, and a less intense class isotropically distributed. The former are likely to be galactic sources, the latter external galaxies.

This classification was originally based on statistics, but it is supported by identifications. The identified objects fall into five groups:

(I) Remnants of supernovae (e.g. the Crab nebula) (4).

(2) Peculiar galactic nebulae (e.g. the Cassiopeia source) (4). 
(3) Peculiar external galaxies (e.g. the colliding galaxies in Cygnus, and M 87) (4).

(4) Normal galaxies (e.g. the Andromeda nebula, and the Clouds of Magellan) (4).

(5) Hil regions (e.g. the Orion nebula) $(5,6)$.

Of these groups, (I) to (3) tend to be of very high intensity at the longer wave-lengths. The characteristic feature appears to be the existence of interstellar gas with very high internal velocities $(\approx$ I000 $\mathrm{km}$. $/ \mathrm{sec}$.). Such high velocities imply enormous energies. Among the identified galactic sources the remnants of supernovae obviously have such energies available. I. S. Shklovsky ( 7 ) has given reasons for supposing that a number of the other sources, for example, that in Cassiopeia, are also remnants of supernovae, but this view has not been accepted by Baade and Minkowski. Similarly in the case of the extragalactic sources the colliding galaxies have fully adequate energy available, but the energy source in other cases is obscure. In this connexion the ratio of energies emitted in the radio and optical parts of the spectrum is of interest. This ratio is of the order of $\mathrm{IO}^{-6}$ overall for our galaxy and some other normal spirals; it is $\mathrm{IO}^{-3}$ for several of the intense sources; and it is actually about unity for the Cygnus source.

Group (5) are observed to be prominent at the higher frequencies (wave-lengths Io and $20 \mathrm{~cm}$.) and it appears that in them the principal emission mechanism is thermal (free-free transitions).

The nature of the mechanism, or mechanisms, responsible for high intensity cosmic radio waves (background and sources) is an outstanding problem of radio astronomy. In this branch of radio astronomy discoveries have tended to pose new problemsmechanisms of origin, the nature of emitting objects, the types of population in which such objects occur-rather than give answers to old ones. When these new questions are answered we can expect many more direct contributions to astrophysics.

The hope of answering these questions depends on the proper merging of facts from three distinct disciplines: on more precise and comprehensive radio observations, on better understanding of radio-wave phenomena in highly rarefied gases, and on optical astronomy. Of these the line of progress of the first is obvious. It is clear that surveys with much greater resolution are required at a number of wave-lengths to give the distribution and the spectra of both discrete sources and background. Further, this is now technically feasible and beginnings have already been made in Cambridge, Sydney and Manchester. But the programme indicated is extensive, and as noted on p. 573 below, co-operative planning between observatories is desirable.

When these questions are answered, what then? First, because with radio waves we can see without obscuration through the galaxy, there should result a wealth of detail on galactic structure. Secondly, we may extend the limits of the observable universe. For example, the Cygnus source, the colliding galaxies, which is at a distance of $3 \times 10^{7}$ parsecs, has a flux density $\mathrm{IO}^{3}$ or $I \mathrm{O}^{4}$ times the threshold for detection. A similar object if it exists would be detectable far beyond the threshold of optical visibility. But so far we lack criteria to determine distances of sources which are not identified optically.

\section{Cosmic radio waves: the $\mathrm{I} 420 \mathrm{Mc}$./sec. hydrogen line}

Observations of this line, unlike those of the continuous spectrum, do not suffer from the handicap that the source is unknown. The line originates in interstellar clouds of neutral atomic hydrogen. Since it permits direct observations for the first time of this form of matter, a form which comprises something like half the mass of the known universe, its importance in astronomy can scarcely be overestimated. And in few branches of astronomy has such spectacular progress been made as that in the three years since the first detection of the line by H. I. Ewen and E. M. Purcell.

The radiation has been studied in some detail in emission from the region near the galactic equator and from the Clouds of Magellan. It is very weak, peak brightness temperatures being commonly from $10^{\circ}$ to $100^{\circ} \mathrm{K}$, and in consequence observations are difficult. The emission is believed to be determined by collisions and so depends on the kinetic temperature of the gas. The natural line-profile is extremely narrow and the 
spread of observed profiles, normally of a few hundred kilocycles per second in width, is due to differing line-of-sight velocities of the contributing matter.

More recently line absorption has been observed (8). This is recognizable where an intense source of continuous radiation lies beyond the absorbing hydrogen. In practice sufficiently intense sources are restricted to the more intense discrete sources of continuous radiation. In such cases it becomes possible to observe both the absorption profile and the emission profile (of the gas in the immediate vicinity). Such information, together with reasonable postulates as to temperature distributions, can lead to firm estimates of the kinetic temperature of the gas and to information about the relative position along the line of sight of the discrete source and the atomic hydrogen.

The first astronomical problem to which $1420 \mathrm{Mc}$./sec. observations have been applied is the delineation of the spiral structure of our galaxy (9). A line-profile in a direction near the galactic equator typically shows several peaks. Each peak is attributed to a concentration of interstellar hydrogen with the line-of-sight velocity corresponding to the frequency-displacement of the peak. Now the principal component of velocity is that due to galactic rotation. Since the rotational characteristics are known, at least approximately, it is possible to deduce the expected line-of-sight velocity for an object at any position. Conversely, applying the concept to the available observations near the galactic plane, the line-of-sight velocity gives the position. Following this idea the concentration of hydrogen causing the peak in the profile can be located. Further observations in different directions indicate that in the vicinity of the galactic plane vast elongated masses of hydrogen extend around the galaxy. These clearly indicate spiral structure, the great gaseous arms presumably delineating also the distribution of the population I stars characteristic of spiral arms. This simple argument can be applied only to regions further from the galactic centre than is the Sun because there are within this distance two alternative positions. Other arguments are being employed to resolve this ambiguity and determine the structure inside.

The current position is that numerous observations taken in Holland and a few in Australia have shown the existence of a number of arms which, in the region external to the solar orbit, have been traced around to the far side of the galaxy. The observed structure is complex and the longer arms, which should best show the gross features, are nearly concentric circles about the galactic centre. In particular the evidence as to whether the spiral is left-handed or right-handed is not yet conclusive. Present opinion is that in relation to the sense of galactic rotation the arms are trailing (as a catherinewheel).

The broad objective of this work is the complete determination of the distribution and motion of interstellar hydrogen within our galaxy. This has obvious implications concerning galactic structure, dynamics, and history. Such a project requires a vast number of observations and, because the desired results do not follow uniquely from the observations, demands a combination of imagination and critical care in interpretation. But a mere three years of observations of the $1420 \mathrm{Mc}$./sec. line has given us a knowledge of the distribution of hydrogen throughout the galaxy comparable with the knowledge of stellar distribution obtained optically throughout the whole history of astronomy.

The second problem which has been studied is the distribution of interstellar hydrogen in the Magellanic Clouds (ro). Here we see the hydrogen in relation not to small details but to whole galaxies. Each of these galaxies is found to be embedded in a vast cloud of hydrogen extending far beyond the bright optical region. In the denser central regions these clouds are about equally dense despite considerable differences in the stellar populations and dust content of the two Clouds. In addition to estimates of the amount of hydrogen present, the observations give line-of-sight velocities from which the rotational characteristics of each of the Clouds have been determined. This leads to estimates of total mass, stars plus gas. Deductions as to detailed structure may be expected when more refined observations are obtained. It is clear that such detailed observations are likely to yield a remarkably complete three-dimensional picture of the structure of these galaxies. 
Successful observations like these suggest the extension of the techniques to many other more distant galaxies. But here serious observational difficulties appear. More distant galaxies are smaller in angular size and demand very large aerials to resolve the individual parts, or even to detect them. So far no other galaxies have been detected. There is little doubt that the larger aerials becoming available, together with improved techniques, will permit detection of a number of the nearer galaxies, but this number is likely to be seriously limited unless sensitivity and resolving power can be considerably increased. Such observations, however, may throw light on one of the classic problems of astronomy, the red-shift of distant galaxies. Observations of the corresponding radiofrequency shift would conclusively test whether this shift accurately follows the Doppler displacement law.

The possibility of the detection of other spectral lines has been suggested by several authors (II), but no successful observations have yet been reported.

\section{The Sun}

The radio emission from the Sun comprises a steady background coming from the whole Sun together with intense localized disturbances with durations ranging from seconds to months. The former component is due to thermal emission from the hot ionized gases of the solar atmosphere and through its study information can be obtained about temperature and density distributions in the solar atmosphere. The mechanism of origin of the disturbed components is still not settled and interpretation of observations in terms of physical conditions in the Sun is consequently handicapped. In addition to observations of solar emission, information about the outer corona has been derived from observations of the occultation of a radio source. The radar or echo method has not yet been applied to the study of the Sun, but is believed to be feasible, though powerful equipment would be required (r2).

The study of the distribution of the background emission requires very high angular resolution, and two elegant techniques $\left(\mathrm{r}_{3}, \mathrm{I}_{4}\right)$ have been applied for obtaining the twodimensional brightness distribution (see pp. 57I below). The results show extension beyond the photosphere and, at decimetre wave-lengths, strong limb-brightening in the neighbourhood of the equator but not at the poles. The marked asymmetry shows that two-dimensional surveys are essential and that one-dimensional observations, for example, eclipse observations at a single station, can be interpreted only with the help of supplementary information. But, in this role, eclipse observations are valuable.

It is not yet known if the background varies throughout the sunspot cycle; the above results apply to sunspot minimum. Corresponding results near maximum will be more difficult to obtain because of the greater difficulty involved in eliminating the effect of the more numerous disturbances.

The radio-brightness distribution over the solar disk at a given wave-length depends on the distribution of electron density and temperature in the solar atmosphere and changes markedly with wave-length owing to the rapid increase of height of origin with increasing wave-length. The actual density and temperature distribution must conform with the distributions of brightness at all wave-lengths, and a model of the solar atmosphere which does not take into account radio data over a wide range of wave-lengths must be seriously open to doubt. At present the available radio data cover a limited wave-length range and it is to be hoped that good observations covering the full range will shortly be taken. The time will then be ripe for the derivation of a reliable model of the solar atmosphere.

The occultation observations have shown that the influence of the Sun extends to far greater distances, about ro solar radii, than had been expected(rs). This influence has been explained in terms of irregularities in the outer corona, a sort of turbidity effect. Such effects have been observed at each of the three occultations for which successful observations were taken, which suggests that turbidity effects are normal.

The emission from disturbed areas may be divided into various classes. We shall 
consider a class characteristic of decimetre wave-lengths, the 'slowly varying component', and a class including various sorts of intense bursts which are typically observed on metre wave-lengths. These disturbances are related in diverse ways with optical solar activity and the relations with flares are particularly interesting.

The slowly varying component is due to the occurrence of highly emitting regions which are closely connected with sunspots and probably even more closely with Ca and $\mathbf{H}$ plages. These regions have diameters of the order of 5 minutes of arc; they have brightness temperatures in the range 0.5 to Io million degrees Kelvin; and they tend to persist for weeks or months. The current explanation of the phenomenon is that the emission is thermal emission from regions in the lower corona of greater than normal density and temperature (Waldmeier's 'coronal condensations'). This explanation is very plausible, but has not yet been subjected to the obvious observational test of comparing optical and radio observations of the same region.

Observations of the intense metre wave-length bursts have been very much curtailed over the past few years by their rarity during sunspot minimum, but with the approaching maximum they should again offer a fruitful field for research. The characteristic features of these bursts are the great intensities (flux densities range up to a million times that of the quiet Sun and brightness temperatures up to $10^{10}$ or even $10^{15} \mathrm{deg} . \mathrm{K}$ ) and the rapid rates of change (durations of the order of seconds or minutes).

These features appear incompatible with an origin in thermal emission from hot gas and two rival theories have been hotly debated, a microscopic and a macroscopic theory. The first considers the burst to be due to incoherent emission from extremely energetic electrons, the second that it is due to groups of electrons moving coherently and emitting in-phase radiation, e.g. plasma oscillations. The recent observational evidence that certain bursts include a fundamental and second harmonic strongly supports the plasmaoscillation interpretation, at least for the bursts concerned (16).

The available observations on bursts are not comprehensive. Thus the size of the emitting area is known to be small with respect to the solar disk, but the sources have not been resolved; the sources of certain bursts (outbursts) have been observed to move rapidly over the solar disk while others, presumed similar, have been shown to have a progressive drift in frequency toward the lower frequencies; some bursts have circular (or elliptical) polarization, certain evidence of magnetic fields, others have random. On the radio side the subject requires simultaneous observations of all the features of particular bursts. The optical side presents a challenge. Is it possible to observe a peculiarity in the light, e.g. in the spectrum, from the actual region in which a radio burst is being generated? The difficulties are twofold. First, a burst is a localized, short-duration, unpredictable phenomenon. Secondly, the region is probably well up in the corona. But if such observations are possible they are likely to be of outstanding importance.

Despite the gaps in information, two most interesting linked hypotheses $(16, x y)$ have been formulated about two classes of burst. One class (the outburst) is thought to be due to the disturbance caused by the passage up through the solar corona with velocities of the order of $1000 \mathrm{~km}$. $/ \mathrm{sec}$. of the clouds of corpuscles responsible for the great magnetic storms and associated aurora. Another class of burst (Wild's 'Type III burst') is supposed to be similarly caused by even faster particles with a velocity of about one-fifth of the velocity of light. Now bursts of these two classes sometimes occur together in association with a solar flare. The further suggestion is made that the two may originate in a common explosive disturbance which gives rise simultaneously to a high-velocity and a low-velocity stream of particles. The high-velocity stream, it is suggested, may have some connexion with the cosmic ray increases observed to accompany certain solar flares.

\section{The Moon}

Radio observations of the Moon have been made, using both of the radio methods, radar and natural emission. The first has not yet given significant information about the Moon though it is potentially capable of giving precise distance measurements. The 
second, utilizing radiation emitted thermally by the lunar surface, has given measurements of the temperature and has led to deductions about a layer of dust overlying the solid surface (r8).

\section{Meteors}

Meteors are detected by radio using the radar method. It is not known if radio waves are also emitted naturally by meteors. As the optical observer sees not the meteoric particle itself but the light from the incandescent gas surrounding it, so the radio observer observes an echo from the associated ionized gas. The study of meteor echoes has led to a considerable clarification of ionization and reflection phenomena. It is also one of the most powerful tools for the determination of the characteristics of the atmosphere in the range of heights where meteors are seen (around roo $\mathrm{km}$.).

Two important features of radio observations of meteors are (i) the observations are not hindered by clouds or daylight and (ii) a sensitivity considerably greater than that of optical equipment is obtainable, leading, for example, to sporadic meteor rates of several hundred per hour. With some elaboration it is possible to obtain details of meteor radiants and velocities, but for individual bright meteors optical observations with cameras with precise timing facilities at two sites appear to give more direct and precise information.

Two important astronomical results have been derived from radio observations. The first is the location of several important meteor streams incident on the daylight side of the earth and consequently unobservable optically (rg). The second is the resolution of the old controversy of whether or not a considerable proportion of sporadic meteors are of interstellar origin ( 19,20$)$. Radio observations have shown definitely that meteors with velocities greater than the velocity of escape from the solar system are either non-existent or very rare. It may be inferred that the vast majority of meteors are members of the solar system.

\section{The ionosphere}

Information about the ionosphere is one of the useful by-products of radio astronomy. Waves from astronomical objects have to pass through the whole terrestrial atmosphere on their way to the observer and are subject to absorption, bending, and scattering to a greater or less extent. Significant effects occur in two regions: in the troposphere for all wave-lengths, and, particularly for longer wave-lengths, in the ionosphere. A region of special interest is the part of the ionosphere above the $\mathrm{F} 2$ maximum of ionization because this region is out of reach of methods using radio signals transmitted from one point on the earth to another.

Refractive bending in the ionosphere is believed to be large for longer wave-lengths and systematic corrections to radio position measurements will be necessary. A study of these corrections is a necessary step in radio astronomy and, conversely, knowledge of this bending is likely to yield worthwhile information about the ionosphere (2r).

Absorption at vertical incidence has been studied at a frequency of $18 \mathrm{Mc}$./sec. and has been shown to be appreciable in both the $\mathrm{D}$ and $\mathrm{F}$ regions (22). It has a regular diurnal and seasonal variation, but shows additional sporadic increases many of which are due to the sudden ionospheric disturbances (S.I.D.'s) associated with solar flares. In fact this method of studying S.I.D. effects may well prove to be the best quantitative method yet devised (23).

The best known of the ionospheric effects is the scintillation of radio stars. There is evidence that in England the effect is due mainly to irregularities in or above the F 2 region of the ionosphere, but in Australia there is evidence for a contribution from sporadic $\mathrm{E}$ also. Consequently in different circumstances the contributing region should be identified before results are interpreted. The scintillations have been applied mainly to the determination of winds or, properly, drifts of the irregularities of ionization in the ionosphere. The pattern of scintillations on the ground is observed to drift in preferred 
directions and from this the corresponding ionospheric drift can be inferred. The irregularities are several kilometres in extent and speeds of the order of $\mathrm{Ioom}$./sec. are common.

A recent observation of Moon echoes promises to provide an outstandingly important ionospheric parameter, viz. the total number of electrons in a column of unit cross-section through the ionosphere (24). Moon echoes were known to fade slowly. This fading has now been shown to be due to the rotation of the plane of polarization of the signals in passing through the ionosphere. The two magneto-ionic components, each nearly circularly polarized, suffer different retardations. On emergence they combine to give a linearly polarized wave whose plane of polarization rotates as the relative retardations change. For the relevant conditions the relative retardation is proportional to the total ionization in the line of sight.

\section{Technical development}

Radio astronomers are continually frustrated in their observations by lack of angular resolution and the most important technical developments are those directed to improving this situation. Other requirements are higher sensitivity and more reliable calibration methods.

\section{Angular resolution}

Angular discrimination in radio astronomy is limited by diffraction considerations similar to those which in optics limit the resolving power of a telescope whose aperture is a given number of wave-lengths. But because the radio wave-lengths are a millionfold greater the limitations are much more stringent. The direct approach to high directivity is to use very large aerials, arrays or parabolic reflectors. Because the structures necessary for the required degree of resolution are so large a number of indirect methods using interference have been developed, but these all have their own limitations. Since the circumstances of the various problems posed by radio astronomy differ, it is desirable to consider the basic problems separately.

Observations of cosmic radio waves pose two problems: general surveys over the sky at various wave-lengths with a resolution of, say, a degree, and more detailed surveys of restricted areas including discrete sources with a resolution of a fraction of a minute of arc.

On the shorter wave-lengths the general survey problem can be met using parabolic reflectors though the required sizes are uncomfortably great, for example, $5^{\circ}$ or roo m. diameter at a wave-length of $I \mathrm{~m}$., but on much longer wave-lengths the construction of adequately large steerable parabolas appears impracticable irrespective of cost.

One aspect of the survey problem is the location of discrete sources and most of our present knowledge of these sources has been obtained with two-element interferometers. This technique is being extended in Cambridge, where four large cylindrical parabolas are used in pairs in a two-element system. Each reflector is $100 \times 12 \mathrm{~m}$. and the total area 4700 sq.m. This instrument has been in operation since I953 and, as noted above, the total number of sources expected is about $\mathrm{I} 700$.

Interferometer records are very difficult to interpret when several sources or any complex distribution of brightness falls within the primary beam. For this reason a pencil-beam device has great advantage. This has been achieved in a recently developed system, the 'Mills Cross' (25). Two long thin aerials are arranged in the form of a cross and the outputs combined alternately with $0^{\circ}$ and $180^{\circ}$ phase difference. The corresponding alternation in output is proportional to the intensity of sources within the region common to the two beams since this is the only received radiation which is phase-coherent. Very long crosses can be constructed with correspondingly high resolution, and the system registers both the discrete sources and the background. In the present Sydney model, which operates at $3 \frac{1}{2} \mathrm{~m}$., each arm is about $500 \mathrm{~m}$. long and the effective beam is a circle of $\frac{3}{4}^{\circ}$ diameter. This model was completed in r 954 .

There are thus three distinct approaches to the cosmic survey problem: the giant steerable parabola as exemplified by the 250 -foot radio telescope under construction at 
Jodrell Bank, the large interferometer as used in Cambridge, and the Mills Cross as used in Sydney. Each has distinct features and a proper assessment of their relative merits will only be possible in the light of the results obtained from each.

Solutions to the problem of surveys of areas minutes of arc in extent have been found in cases where the area is an outstanding source (intense and isolated from comparable neighbours $(26,27))$. Two-aerial interferometers were used with a series of spacings along each of a series of differently oriented lines. Spacings up to $10 \mathrm{~km}$. were used and it is apparent that the methods are very cumbersome. In the case of weaker sources the difficulties would be increased because larger, more directive, aerials would be required. It is clear that the problem is really exacting and that an elegant solution would be a major advance.

The Sun presents rather different problems. Its diameter is half a degree so that, to be useful, details are required to a minute of arc or so. A simple aerial (array or parabola) with a resolution of, say, $3 \mathrm{~min}$. of arc must, at $\mathrm{I} \mathrm{m}$. wave-length, be $\mathrm{r} \mathrm{km}$. in extent and, even at $20 \mathrm{~cm}$., $200 \mathrm{~m}$. These dimensions are so great that simple aerials are very severely limited in applications involving details of distribution.

At short (decimetre and centimetre) wave-lengths the Sun normally presents a complex pattern of brightness making high resolving power essential. But fortunately the pattern normally persists for at least a day or so and the Sun is outstanding against the galactic background. Observations of this complex distribution in two dimensions have been made in two ways: by multiple-spacing, two-aerial interferometers (28) and with multipleelement interferometers (29). Each method yields the one-dimensional brightness distribution (strip-wise scanning) for a particular orientation. Observations are repeated for different orientations and the results combined by a method described by O'Brien to obtain the two-dimensional picture. Such observations show a complex background due to the quiet Sun together with transitory bright areas due to disturbances. In interpretation difficulties are met in separating these two components. The multiple-element interferometer which scans the Sun with a knife-edge beam has the merit of giving a direct indication of bright patches.

It is clear that the real requirement for this work is a system giving a 'picture' of the Sun at the operating wave-length such as would be given by a pencil-beam scanning technique. Such a system was proposed by W. N. Christiansen at the U.R.S.I. General Assembly in r954. He suggested combining two multiple beam interferometers in the manner of a Mills Cross. Each of the individual arms gives a series of parallel knife-edge beams, the two lying in perpendicular directions. The combination would respond to the regions common to the two systems thus giving effectively a series of pencil beams arranged in a rectangular grid. The placing of these beams could be such that the Sun in its passage through the sky was scanned, television-fashion, by one beam at a time. Such a system is inherently capable of giving daily 'radio-heliograms'.

At metre wave-lengths the typical disturbances tend to be of seconds or minutes duration so that very rapid observations are required. Fortunately, however, many of them are very intense and tend to occur one at a time so that the burst overshadows both the galactic and solar backgrounds.

Three types of measurement have been informative: measurements of dynamic spectra, of position, and of polarization. Equipment for spectra has been developed in Sydney by J. D. Murray and J. P. Wild, but the more recent developments have not yet been described. Rapid measurements of position and polarization at a single frequency were made by A. G. Little and Ruby Payne-Scott (3o), using a two-aerial sweptlobe interferometer. A new form of interferometer developed by J. P. Wild and J. A. Roberts was described by J. L. Pawsey at the U.R.S.I. General Assembly in I954. In this form the necessary phase change is produced in a fixed two-aerial system by rapidly sweeping the frequency over a range of two to one. The method has not yet been applied to solar bursts but when applied should give positional information over a range of frequencies. 


\section{Receivers}

Receivers used in radio astronomy are required to measure exceptionally small power levels and must be correspondingly sensitive. Sensitivity depends on minimizing the noise generated internally and on suppressing fluctuations in output due to such causes as gain fluctuations. No marked progress in the first is evident.

The main line of attack on the latter has been through rapid comparison systems, either in the original form (3r) in which the noise from the aerial is compared many times a second with that from a resistor at ambient temperature (a dummy aerial) or in the modified form (32) in which the dummy aerial includes a diode whose noise output is automatically equalized with that of the aerial. Work is also proceeding on attempts to produce receivers which are sufficiently stable to obviate the necessity for such devices. An important factor favouring such a development is that the inherent noise fluctuations (due to the random nature of the input) are theoretically only half as great in the direct system and the ultimate limit to sensitivity consequently twice as great for the same integration time. The present position is that rapid comparison systems can be made whose freedom from fluctuations approaches the theoretical limit; direct receivers can similarly give nearly ideal results for short periods of time. But each requires considerable care in setting up and in operation. Relatively fool-proof receivers would be a useful development.

Phase-switching, another variant of the comparison technique, was first used by M. Ryle (33) in a two-aerial interferometer. The two aerials are connected to the receiver with either $0^{\circ}$ or $180^{\circ}$ phase difference, the connexion alternating rapidly between the two conditions. The output is switched synchronously and the average difference between the outputs for the respective connexions is recorded. The output shows the required interference pattern independently of the background. Further, as with the Dicke system, receiver gain fluctuations are suppressed and also certain types of interference. A variant employing electronic switching was developed by Mills who also applied it in his 'Cross' system.

\section{Calibration}

Calibration of radio astronomy equipment usually involves separate measurements of aerial characteristics and of receiver sensitivity. The first is difficult, but no general rules about procedure can be formulated except to suggest as many cross checks as possible. Receiver sensitivity is commonly measured using either a saturated diode, a gas discharge noise source, or hot and cold resistors. It is all too common to find diodes giving incorrect results and it is emphasized that either diodes or gas discharge tubes, which are thoroughly useful as sub-standards, should be checked using the hot and cold resistor technique.

It is clearly desirable that methods for overall calibration should be available and there is a requirement for the development of reliable techniques for this. A step in this direction which has not been exploited to the extent it deserves involves the use of standard cosmic sources for intercomparison purposes: discrete sources as standards of flux density, uniform areas in the sky for radio brightness. It is desirable that the I.A.U. encourage this procedure by deciding on and recommending a suitable sequence of standard radio sources and areas in the sky (see p. 59o below).

\section{International Collaboration}

Radio astronomy was pioneered by radio engineers and physicists rather than by astronomers and as a result another international union, the Union Radio Scientifique Internationale (U.R.S.I.) is vitally interested. In fact Commission V of U.R.S.I. and Commission 40 of the I.A.U. have almost identical interests and a large proportion of common members. This may be illogical, but it is not clear that any good would be done by an attempt at rationalization. The good feature of the current arrangement is that radio astronomers who are able to attend meetings of both unions and so mix with both 
radio research workers and astronomers. It seems best to carry on the present arrangement, but to attempt progressively to channel basically astronomical questions to the I.A.U. and radio ones to U.R.S.I.

The items which follow are ones in which international collaboration may be required and which should be considered by the I.A.U.

\section{Terminology and units}

U.R.S.I. and the I.A.U. (34) have each considered this question, and U.R.S.I. has approved a report (Appendix I) which gives matters on which substantial unanimity was reached. It is recommended that the I.A.U. should consider this report and, if in agreement, should endorse it. It would of course be in order for the I.A.U. to recommend amendments or additions. It may be worth noting that a point which was discussed by U.R.S.I. but rejected because of lack of unanimity was the introduction of a short term for the rather clumsy m.k.s. unit of flux density (watts $\mathrm{m}^{-2}(\mathrm{c} / \mathrm{s})^{-1}$ ). The term 'jansky' was considered as commemorating the founder of radio astronomy. The other important unit, that of brightness (watts $\mathrm{m}^{-2}(\mathrm{c} / \mathrm{s})^{-1}$ steradian ${ }^{-1}$ ) would have become 'jansky per steradian'.

\section{Recognition and designation of discrete sources}

The early surveys of discrete sources of cosmic radio waves suffered from considerable inaccuracy, and when the areas of two surveys overlap the weaker sources do not show a one-to-one correspondence. This is probably due to confusion between complex groups of sources, some of the positions shown being 'blends' of several sources, and the blending process fortunately being different in the different surveys. This confusion should ultimately be resolved by higher resolution surveys, but there are at present a moderate number of reliably known sources. Which of these should be so considered is only known to those working in the field. In consequence the publication of a list of reliably known sources was considered by U.R.S.I., and because the question is of astronomical rather than radio interest, referred to the I.A.U. The President of Commission 40 accepted the suggestion, and with the help of an expert committee had a list (35) prepared. It is reproduced in Appendix 2. It is recommended that the question of issuing further lists in the future should be considered when further surveys become available.

In this list a new system of catalogue numbers for sources has been adopted. This is explained in the preamble to the list. Individual workers should, as in the past, use their own systems for newly discovered sources. They would create confusion if they independently adopted this system for new ones.

\section{Selected areas and discrete sources for standardization of measurements}

Radio measurements of flux density and brightness are notoriously difficult and it is certain that greater use should be made of intercomparison with other cosmic sources. To facilitate flux density measurements of both solar and cosmic sources, it is recommended that the I.A.U. should prepare a list of standard discrete sources; and similarly for brightness, a list of standard areas in the sky. The latter should be selected separately for continuous cosmic emission and for the $1420 \mathrm{Mc}$./sec. hydrogen line as the technical requirements differ.

\section{International planning of research programmes}

In the course of its history the I.A.U. has arranged a great many co-operative observations. Some of the results of these have been excellent in giving more complete information, others have hampered research by suppressing individual initiative through the burden of a vast programme of routine observations. It is essential to consider the pros and cons of co-operative research in relation to each individual project.

There are two types of problem in which international planning can be peculiarly 
useful: ones in which the whole picture can only be seen from the combination of observations taken in different parts of the Earth, and those which are so tedious that the work can only be completed in a reasonable time if it is shared between a number of observatories.

In radio astronomy there is already one co-operative project, the continuous recording at different longitudes of the intensity of solar radio waves on a number of wave-lengths. This project is organized through a sub-committee of U.R.S.I.-Chairman A.H. de Voogtand the results are edited by S. F. Smerd and published in the Quarterly Bulletin on Solar Activity. In this case the results not only have application to solar physics but the information on solar disturbances may be useful in radio communication. Since the project is operating it would appear unnecessary for the I.A.U. to take any action unless members should wish to criticize the plan or recommend modifications.

Another subject which requires consideration is the determination of the detailed distribution and spectra of cosmic radio waves over the sky (requiring observations from both northern and southern observatories). Because of the lack of standardization in techniques it would appear desirable to hold discussions on the type of information required and to leave the detailed planning to informal discussion. A suggestion for the requirements of such surveys is for contour maps showing detail to at least I degree over the whole sky at frequencies at intervals of 2 or 3 to $I$. These should range from the lowest feasible (e.g. Io or $20 \mathrm{Mc}$./sec.), to at least I420 Mc./sec. (e.g. 20, 40, 80, I60, 300, $600,1420 \mathrm{Mc} . / \mathrm{sec}$.). Note that a frequency in the immediate vicinity of $1420 \mathrm{Mc}$. $/ \mathrm{sec}$. is required for interpretation of hydrogen line observations. Planning is required so that northern and southern surveys should be made on similar frequencies with sufficient overlap for mutual checking.

The need for co-operative planning is especially evident in investigations such as the scintillation of radio stars, ionospheric absorption, and the rotation of the plane of polarization of Moon echoes. These matters are of interest to I.A.U. members, but it would appear best, in view of their being of geophysical rather than astronomical interest, to refer them to U.R.S.I. should any formal action be required.

\section{Bibliographical Notes}

A comprehensive bibliography of radio astronomy (exclusive of meteors) under the title Bibliography of Extraterrestrial Radio Noise (including abstracts) has been prepared by Martha E. Stahr-Carpenter and was issued as a part of the report of Commission V of U.R.S.I. (I950). Supplements are issued from time to time. Those interested in obtaining this most useful reference work should communicate with the editor (address: Dept. of Astronomy, Cornell University, Ithaca, New York, U.S.A.).

Important papers on radio astronomy are frequently found outside the normal astronomical journals, particularly in the Australian Journal of Physics (formerly Australian Journal of Scientific Research, A), Comptes Rendus (Paris), Comptes Rendus de l'Académie des Sciences de l'U.R.S.S. (Doklady Akademii Nauk, S.S.S.R.), Nature, Proceedings of the Physical Society, Proceedings of the Royal Society, Philosophical Magazine and Physical Review.

Brief reports of the work of radio observatories in British countries are given each year in Monthly Notices of the Royal Astronomical Society with those of other observatories.

The following are important books and recent integrating papers in radio astronomy:

\section{Books}

B. Lovell and J. A. Clegg, Radio Astronomy (London: Chapman and Hall, 1952. 227 pp.). [This book has been translated into Russian.]

J. L. Pawsey and R. N. Bracewell, Radio Astronomy (Oxford, 1955. 36I pp.).

I. S. Shklovsky, Radio Astronomy (Popular Outline) (Moscow Government Publisher of Technical and Theoretical Literature, 1955, 294 pp.). 


\section{Integrating articles}

W. Dieminger and H. Siedentopf, 'Radioastronomie' (in three parts), Archiv der Elektrischen Ubertragung, 7, 421-7, 507-17, and 555-60 (1953).

International Scientific Radio Union (U.R.S.I.):

Special Report No. 3 on Discrete Sources of Extra-terrestrial Radio Noise (Brussels, General Secretariat of U.R.S.I., I954. $5^{6} \mathrm{pp}$.). [Prepared by a sub-committee consisting of J. G. Bolton, Chairman, F: G. Smith, R. Hanbury Brown, and B. Y. Mills.]

Special Report No. 4 on the Distribution of Radio Brightness on the Solar Disk (Brussels, General Secretariat of U.R.S.I., I954. $4^{6}$ pp.). [Prepared by a sub-committee consisting of W. N. Christiansen, Chairman, J. P. Hagen, A. Hewish, M. Laffineur, and F. G. Smith. Bound with Special Report, no. 5.]

Special Report No. 5 on Interstellar Hydrogen (Brussels, General Secretariat of U.R.S.I., 1954. $26 \mathrm{pp}$.). [Prepared by a sub-committee consisting of J. H. Oort, Chairman, J. L. Pawsey, and E. M. Purcell. Bound with Special Report, no. 4.]

A. C. B. Lovell et al., 'Radio Astronomy', Occasional Notes, R. Astr. Soc. 3, no. I6 (1954), 29-79.

J. L. Pawsey and S. F. Smerd, 'Solar Radio Emission', pp. 466-53r, in The Sun, ed. G. P Kuiper (Chicago Univ. Press, 1953).

Max Waldmeier, Radiowellen aus dem Weltraum (1953. $7^{8} \mathrm{pp}$.). [Reprinted from Viertel jahrsschrift der Naturforschenden Gesellschaft in Zürich, 98, no. 4.]

J. P. Wild, 'Techniques for Observation of Radio-frequency Radiation from the Sun', pp. 676-92 and 734-7 in The Sun, ed. G. P. Kuiper (Chicago Univ. Press, 1953).

J. P. Wild, 'Observational Radio Astronomy', pp. 299-362 in Advances in Electronics (New York: Academic Press, vII, I955).

\section{J. L. PAwsey \\ President of the Commission}

\section{REFERENCES}

(1) H. Alfvén and N. Herlofson, Phys. Rev. 78, 616, 1950.

(2) For example, I. S. Shklovsky, Astr. J. U.S.S.R. 30, 15, 1953.

(3) B. Y. Mills, Aust. J. Sci. Res. A, 5, 266, 1952.

(4) W. Baade and R. Minkowski, $A p . J$. 119, 206 and 215, 1954.

(5) F. T. Haddock, C. H. Mayer and R. M. Sloanaker, Ap. J. I19, 456, I954.

(6) J. P. Hagen, E. F. McClain and N. Hepburn, Proc. Instn Radio Engrs, 42, 181 I, 1954.

(7) I. S. Shklovsky, Astr. J. U.S.S.R. 31, 483, I954.

(8) D. R. W. Williams and R. D. Davies, Nature, Lond., I 73, I I82, I954. J. P. Hagen, A. E. Lilley and E. F. McClain, N.R.L. Report, 4448.

(9) H. C. van de Hulst, C. A. Muller and J. H. Oort, B.A.N. 12, no. 452, I I7, I954.

(ro) F. J. Kerr, J. V. Hindman and B. J. Robinson, A ust. J. Phys. 7, 297, 1954.

(I I) For example, I. S. Shklovsky, C.R. Acad. Sci. U.R.S.S. 97, 25, 1953.

(12) F. J. Kerr, Proc. Instn Radio Engrs, 40, 660, 1952.

(13) P. A. O'Brien, Mon. Not. R. Astr. Soc. II3, 597, 1953.

(I4) W. N. Christiansen and J. A. Warburton, Observatory, 75, 9, I955.

(15) K. E. Machin and F. G. Smith, Nature, Lond., 170, 319, 1952; further English results will be given in a paper by $\mathrm{A}$. Hewish and Russian results by V. V. Vitkevitch.

(I6) J. P. Wild, J. D. Murray and W. C. Rowe, Aust. J. Phys. 7, 439, I954.

(17) J. P. Wild, J. A. Roberts and J. D. Murray, Nature, Lond., 173, 532, 1954.

(18) J. H. Piddington and H. C. Minnett, Aust. J. Sci. Res. A, 2, 63, 1949.

(19) B. Lovell and J. A. Clegg, Radio Astronomy (London: Chapman and Hall, 1952).

(20) D. W. R. McKinley, $A p . J . \times 1 \times 3,225,195 \mathrm{I}$.

(2I) F. G. Smith, J. Atmos. Terr. Phys. 2, 350, 1952.

(22) A. P. Mitra and C. A. Shain, J. Atmos. Terr. Phys. 4, $204,1953$.

(23) C. A. Shain and A. P. Mitra, J. Atmos. Terr. Phys. 5 316, 1954. 
(24) W. A. S. Murray and J. Hargreaves, Nature, Lond., r73, 944, 1954.

(25) B. Y. Mills and A. G. Little, A ust. J. Phys. 6, 272, 1953.

(26) B. Y. Mills, Aust. J. Phys. 6, 452, 1953.

(27) R. Hanbury Brown and R. Q. Twiss, Phil. Mag. 45, 663, I954.

(28) P. A. O'Brien, Mon. Not. R. Astr. Soc. 113, 597, 1953.

(29) W. N. Christiansen and J. A. Warburton, Observatory, 75, 9, 1955.

(3o) A. G. Little and R. Payne-Scott, Aust. J. Sci. Res. A, 4, 489, I95I.

(3I) R. H. Dicke, Rev. Sci. Instr. 17, 268, 1946.

(32) M. Ryle and D. D. Vonberg, Proc. Roy. Soc. A, 193, 98, 1948.

(33) M. Ryle, Proc. Roy. Soc. A, 21x, 351, 1952.

(34) Trans. I.A.U. 8, 613, 1952.

(35) J. L. Pawsey, $A p . J$. 121, I, 1955.

APPENDIX I

\section{U.R.S.I., SUB-COMMISSION VB ON TERMINOLOGY AND UNITS, ELEVENTH GENERAL ASSEMBLY, I954}

We recommend that the following recommendations should be submitted to the I.A.U. for their consideration:

\section{Power \\ Specification of power and polarization of extraterrestrial radiation}

The quantity of incident power shall be specified in terms of the whole radiation received at the point of measurement, per unit area perpendicular to the direction of propagation and per unit of bandwidth at the midfrequency of the received band.

For sources of small angular extent relative to the angular resolution of the observing equipment, the quantity of power is the 'flux density, $(S)$ ', which is measured in watts per square metre per cycle of bandwidth, $(w)(m)^{-2}(\mathrm{c} / \mathrm{s})^{-\mathbf{1}}$.

For extended sources, the quantity of power is the 'surface brightness, $(B)$ ', which is measured in watts per square metre per cycle of bandwidth per steradian, (w) $(\mathrm{m})^{-2}(\mathrm{c} / \mathrm{s})^{-1}(\text { sterad })^{-1}$.

In the case of spectral line emission, the total power in the full frequency range of a line-profile in excess of any continuum is, for sources of small angular extent, the 'line integrated flux density, $\left(S_{\text {int }}\right)$ ' and, for extended sources, the 'line integrated surface brightness, $\left(B_{\text {int }}\right)$ '.

It is undesirable to use the term 'intensity' which, because of multiple uses, is now ambiguous.

Since the total quantity of incident power often is not measured, the procedure used in obtaining it should be stated, for example, for randomly polarized radiation, the single measured quantity is doubled.

\section{Polarization}

The expression 'plane of polarization' is ambiguous and should be avoided. In cases where the polarization of the measurement is significant, the polarization must be specified in terms of the electric vector; and the fact that it is the electric vector must be stated explicitly.

The sense of rotation of a circularly or elliptically polarized wave is defined as 'righthanded' if the electric vector in a fixed plane in the wave-front rotates clockwise when viewed in the direction of propagation, i.e. with the source behind the observer. The opposite sense of rotation is 'left-handed'.

\section{Equivalent temperature formulations}

Formulations employing 'equivalent temperatures' are useful since calibration procedures are generally based in some way on actual temperatures or on temperature concepts. This does not imply that the power considered is necessarily of thermal origin. 
The surface brightness $(B)$ of thermal radiation from a black body of uniform temperature $(T)$ is known from Planck's law. Hence this temperature, under the term 'brightness temperature $\left(T_{b}\right)$ ', may be used as a measure of $B$.

In the case of solar radio waves, the flux density $(S)$ can be specified, without observation of the brightness of individual parts of the Sun, in terms of the "apparent disk temperature, $\left(T_{d}\right)^{\prime}$ of the Sun, i.e. the temperature of a black body having the same angular size as the photospheric disk at the time of the observation, which would yield the observed flux density.

The available power $(P)$ from an aerial, i.e. the power delivered to a matched load per unit frequency band, can be specified in terms of the temperature $\left(T_{a}\right)$ of a uniform temperature enclosure surrounding the aerial, whose thermal radiation leads to the same power per unit frequency band at the terminals. The temperature $\left(T_{a}\right)$, where $P=k T_{a}$, is called 'the equivalent aerial temperature'.

\section{Logarithmic measurement of power}

It is sometimes convenient and concise to use a logarithmic measurement and an agreed zero level when tabulating and discussing observed incident powers. For flux density, the preferred zero level is one watt per square metre per cycle of band-width at the midfrequency of the received band. The preferred logarithmic expression of the ratio of a flux density $(S)$ to this zero level is the 'strength, $(\sigma)^{\prime}$ ', which is the logarithm to the base ten of $S$. For example, if

then

$$
\begin{gathered}
S=2 \times \mathrm{IO}^{-23} \\
\sigma=0 \cdot 3-23 \cdot 0=-22 \cdot 7 .
\end{gathered}
$$

\section{APPENDIX 2}

\section{A CATALOGUE OF RELIABLY KNOWN DISCRETE SOURCES OF COSMIC RADIO WAVES*}

Observations of discrete sources of cosmic radio waves (sometimes called radio stars) are severely limited by the relatively poor resolving power of radio instruments. The more intense or more favourably situated ones can be located with certainty and precision, but there remains a large number whose positions are known very imperfectly. Indeed, where a number of sources are close together the resulting confusion can lead to an assigned position where no source exists, the observation referring to a 'blend' of several sources each slightly removed from the assigned position. In these circumstances it is most difficult for an astronomer who has no direct access to the radio observers to know what certainty to attach to a published location of a radio source.

In an attempt partly to overcome this difficulty it was suggested by the radio astronomy commission of the Union Radio Scientifique Internationale (U.R.S.I.) that the corresponding commission (Commission 40) of the International Astronomical Union (I.A.U.) should prepare and publish a list of sources whose existence was certain, and should supplement this list from time to time as better observations became available.

The members of Commission 40 considered this suggestion sound and have taken steps to prepare an initial list which is given below.

The list was prepared on the invitation of the President of Commission 40 by a committee consisting of J. G. Bolton (Sydney) (convener), R. Hanbury Brown (Manchester), F. G. Smith (Cambridge) and B. Y. Mills (Sydney). These men were selected because they include representatives of each of the laboratories which has published major contributions to this subject. Further, they were already engaged in the preparation of a special report for U.R.S.I. on the discrete sources.

\footnotetext{
* Published in the Astrophysical Journal, r21, 1, January 1955.
} 
It will be noted that different observers have given their own catalogue numbers to particular sources. In an attempt to gain a measure of uniformity, a new designation headed 'I.A.U. number' has been allotted to each source. This system is based on position in the sky, the five symbols being allocated as indicated below.

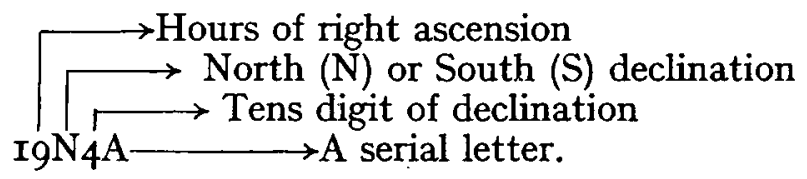

At present the major sources are well known by their constellation names, e.g. Cygnus and Cassiopeia, and there is every reason to expect this will continue. Catalogue numbers are essential for the minor sources.

It is hoped to publish supplementary lists of sources from time to time.

In conclusion I wish to express the thanks of the members of Commission 40 to those who have contributed to the compilation and checking of this list: to the members of the committee, named above, and also to J. P. Hagen, J. D. Kraus, R. Minkowski and M. Ryle who helped in the checking.

J. L. Pawsey

\section{I.A.U. Catalogue of Discrete Sources of Cosmic Radio Waves (I954)}

This short catalogue is divided into two lists. List I contains those sources whose existence has been definitely established, accurate positions given by a number of observers and for which optical identifications have been secured. In some cases the identifications are supported by agreement between the radio and optical sizes.

List 2 contains sources for which the radio positions are not known so accurately. Their inclusion is considered justified because either a reasonable identification has been suggested for the source (List $2 a$ ) or there is good agreement between the positions and flux densities given in surveys by two or more independent observers. The catalogue numbers of the independent observers have been given with a prefix identifying the survey, as follows:

BSS Bolton, Stanley and Slee (13).

R Ryle, Smith and Elsemore (8).

HB Hanbury Brown and Harard (ir).

M Mills (z).

NRL (Naval Research Lab.) Haddock, Mayer and Sloanaker(18).

$\mathrm{S} \quad$ Shain and Higgins (14)

References alongside data in the list denote the sources of the data, explained in a key at the end. Where the uncertainty in the observational data can be indicated simply, it has been done, for example, in the measured positions of the sources.

The catalogue was compiled early in 1954 on the basis of observations published at that time. More detailed and reliable observations are rapidly becoming available as better equipment is put into operation. Such unpublished results are not included in the catalogue but have been used to check the reality of the great majority of those sources included. In this respect data supplied by J. P. Hagen and J. D. Kraus, and preliminary observations obtained by B. Y. Mills have been particularly helpful. 


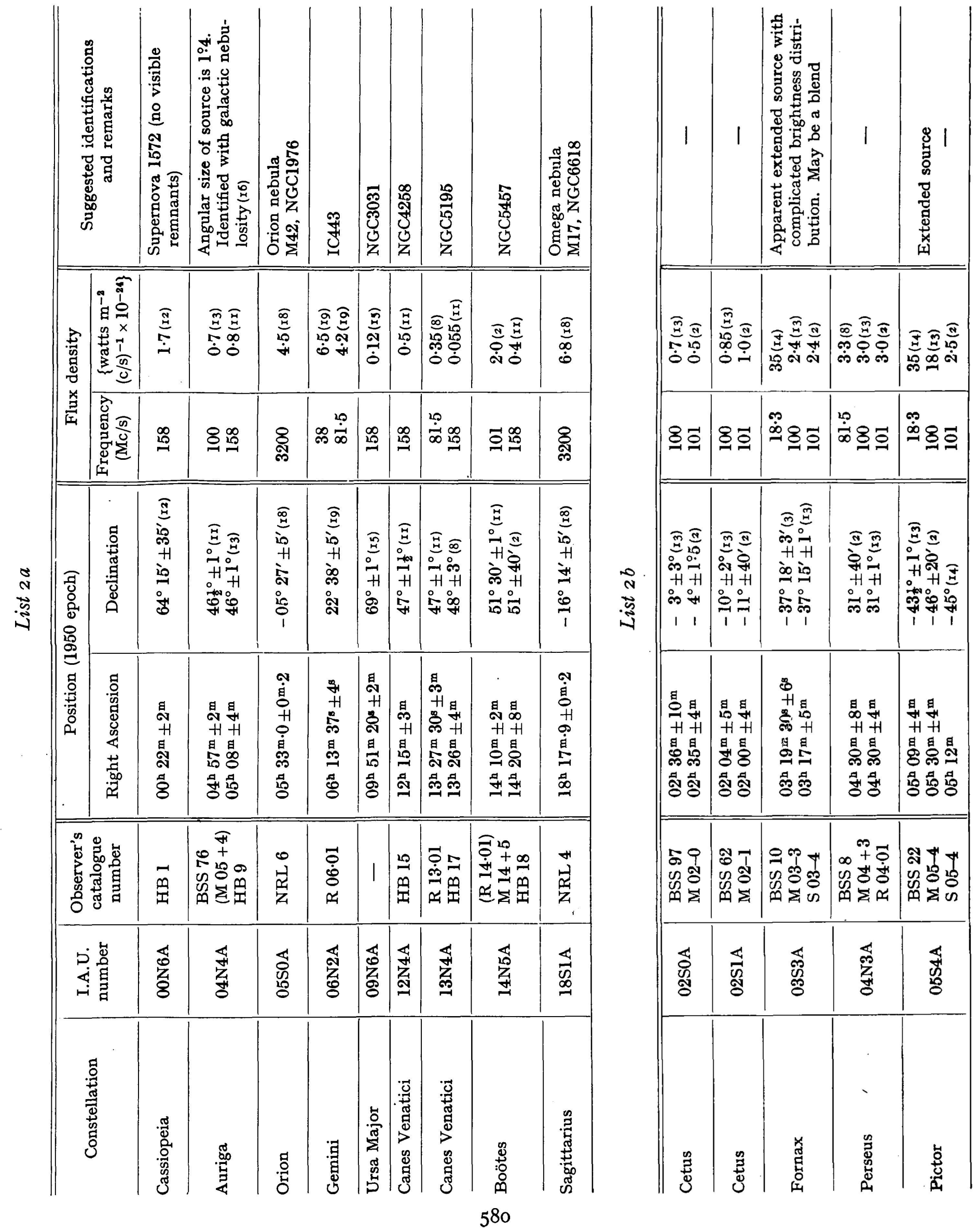


(I) G. J. Stanley and O. B. Slee, Aust. J. Sci. Res. A 3, 234, 1950.

(2) B. Y. Mills, Aust. J. Sci. Res. A 5, 266, 1952.

(3) B. Y. Mills, A ust. J. Sci. Res. A 5, 456, 1952.

(4) F. G. Smith, Nature, Lond., 168, 555, 1951.

(5) R. Hanbury Brown, R. C. Jennison and M. K. Das Gupta, Natuve, Lond., 170, ro61, 1952.

(6) J. G. Bolton, K. C. Westfold, G. J. Stanley and O. B. Slee, A ust. J. Phys. 7, no. r, 96, I954.

(7) F. G. Smith, Nature, Lond., 170, 1065, 1952.

(8) M. Ryle, F. G. Smith and B. Elsmore, Mon. Not. Roy. Astr. Soc. 110, 508, 1950.

(9) J. H. Piddington and H. C. Minnett, Aust. J. Sci. Res. A 5, 17, 1952.

(Io) B. Y. Mills, A ust. J. Phys. 6, no. 4, 452, 1953.

(II) R. Hanbury Brown and C. Hazard, Mon. Not. Roy. Astr. Soc. 113, 123, 1953.

(12) R. Hanbury Brown and C. Hazard, Nature, Lond., 170, 364, 1952.

(13) J. G. Bolton, G. J. Stanley and O. B. Slee, Aust. J. Phys. 7, I ro, 1954.

(14) C. A. Shain and C. S. Higgins, A ust. J. Phys. 7, no. 1, 130, 1954.

(I5) R. Hanbury Brown and C. Hazard, Nature, Lond., 172, 853, 1953.

(I6) R. Hanbury Brown, H. P. Palmer and A. R. Thompson, Nature, Lond., x73, 945, I954.

(17) J. E. Baldwin and B. Elsmore, Nature, Lond., 173, 818, 1954.

(18) F. T. Haddock, C. H. Mayer and R. M. Sloanaker, Ap. J. Ir9, 456, x954.

(19) J. E. Baldwin and D. W. Dewhirst, Nature, Lond., 173, 164, 1954.

(20) J. H. Piddington and H. C. Minnett, Aust. J. Sci. Res. A 4, 459, 1951.

(21) R. X. McGee and J. G. Bolton, Nature, Lond., 173, 985, 1954.

\section{APPENDIX 3}

\section{ABSTRACTS OF PAPERS ON RADIO ASTRONOMY IN THE U.S.S.R.*}

V. V. Vitkevitch (C.R. Acad.Sci. U.R.S.S. 77, no. 4, 195I) advanced a new method for the investigation of the solar corona consisting in the study of radio waves, emitted by localized sources, passing through the corona.

V. V. Vitkevitch (in the Press), in analysing the observations of the radio emission from the Crab nebula carried out in $1952-54$ at two different interferometer spacings, showed that the outer regions of the solar corona create an appreciable scattering of radio waves (in the metre range) at a distance of $12-15$ solar radii from the photosphere. The instability of the interference pattern showed the presence of dynamical processes in the solar corona at a distance of ro-I 5 radii from the photosphere. These processes can be explained by motions of electron condensations with velocities of the order of either $10^{5} \mathrm{~km}$. $/ \mathrm{sec}$., or IO $\mathrm{km}$. $/ \mathrm{sec}$.

V. V. Vitkevitch (A.J. U.S.S.R. 29, no. I, 1952) investigated the sensitivity of radio telescopes for reception of radio emission from various cosmic sources.

V. V. Vitkevitch and R. L. Sorotchenko (A.J. U.S.S.R. 30, no. 6, 1953) suggested a new type of radio telescope, consisting of a number of aerials. The system has a number of narrow lobes in the directional diagram, distributed at various angular distances.

V. V. Vitkevitch (C.R. Acad. Sci. U.R.S.S. 86, no. I, I953) advanced a new system of radio reception for the determination of the co-ordinates of bursts on the Sun. The system consists of an interferometer with two receivers, connected at intervals of a quarter of a wave-length.

V. V. Vitkevitch (A.J. U.S.S.R. 29, no. 4, I952) developed the theory of an interference method of radio reception and calculated the influence of the angular diameter of a source, asymmetry of aerials and the width of the transmission band upon the decrease of the depth of modulation in an interference pattern.

V. V. Vitkevitch (A.J. U.S.S.R. 29, no. 5, 1952) proposed a radiometer with frequency modulation for reception of monochromatic cosmic radio emission and calculated the sensitivity

* These abstracts were prepared by I. S. Shklovsky at the end of 1954 for the President to assist him in the preparation of his report, but were delayed in transit. Because the material is substantial and is not readily available to many astronomers it is appended here. 
of such a system. He suggested determining the distance of local sources by establishing their intensity for $21 \mathrm{~cm}$. wave-length.

V. V. Vitkevitch (C.R. Acad. Sci. U.R.S.S. 9I, no. 6, I953) elaborated a new method of spatial selective reception of radio emission from cosmic sources, based upon reception in a wide range of frequencies. This method can be used for the construction of a telescope of super-high resolving power.

Observations of radio emission from the Sun during the total solar eclipse of 25 February I952 permitted V. V. Vitkevitch to discover the effect of an increase of brightness on the limb (in the Press).

The first decimetre radiometer for $50 \mathrm{~cm}$. waves was constructed by V. V. Vitkevitch for reception of radio emission from cosmic sources. The radiometer possesses an original system of co-axial modulator and is, at the same time, a differential radiometer, measuring the difference of energies of two sources of any kind. (A paper has been prepared.)

V. V. Vitkevitch suggested and realized an interference method for observation of the radio emission from the Sun in the $50 \mathrm{~cm}$. range during an eclipse. More detailed data concerning the distribution of the brightness of the solar disk were obtained. The effect of an increase of brightness at the limbs was not observed. (A paper is being prepared.)

V. V. Vitkevitch discovered, during systematic interference observations of radio emission of the Sun in the $50 \mathrm{~cm}$. range carried out in $195 \mathrm{I}$ and 1952 , that the solar corona has in the first approximation the form of an ellipse with the axis turned in the direction of the equator (prepared for Press).

V. V. Vitkevitch discovered in 1953, during his regular observations of the radio emission of the Sun in the $10 \mathrm{~cm}$., $50 \mathrm{~cm}$. and $3.5 \mathrm{~m}$. ranges, that the position of the centre of radio measurements for these wave-lengths shows an appreciable deviation from one day to another. On $10 \mathrm{~cm}$. and $50 \mathrm{~cm}$. waves such a deviation often reaches $5^{\prime}$ and on $3.5 \mathrm{~m}$. waves $10^{\prime}$ to $\mathrm{I} 5^{\prime}$. A relation of these deviations in the position of the centre of radio emission to the astrophysical characteristics of the Sun was not established (prepared for Press).

V. V. Vitkevitch and B. M. Chikhatchev carried out observations of the radio emission of the Sun during the total eclipse of the Sun on 25 February 1952 in the ranges of $2.6 \mathrm{~m}$. and $2 \mathrm{~m}$. Data concerning the distribution of intensity of radio emission from the solar disk in the same ranges were also obtained. During the total phase the remaining emission equalled $50 \%$ for $2.6 \mathrm{~m}$. waves and for $2 \mathrm{~m}$. waves $42 \%$ (in the Press).

B. M. Chikhatchev carried out observations of the radio emission of the Sun during the total solar eclipse of 25 February $195^{2}$ on wave-lengths of $1 \cdot 5 \mathrm{~m}$. and I $\mathrm{m}$. and obtained data concerning the distribution of the intensity on the solar disk. During the total phase the remaining emission on $\mathrm{I} \cdot 5 \mathrm{~m}$. waves constituted $36 \%$ and on $\mathrm{I} \mathrm{m}$. waves $33 \%$ (in the Press).

V. V. Vitkevitch carried out simultaneous observations of the 'small peaks' of radio emission of the Sun for two close frequencies of 205 and $209 \mathrm{Mc}$. /sec. It was established that some 'small peaks' possess a narrow spectrum of the order of $6 \mathrm{Mc}$. $/ \mathrm{sec}$., which corresponds to a relative width of the emission spectrum of the order of 0.03 (in the Press).

V. V. Vitkevitch discovered during observations of the radio emission of the Sun on 26 September 1952 a new type of burst of solar radio emission. The temporary increase of a burst suggests two branches-a branch for the long and a branch for the short wave-lengths. The bursts in the range of $\mathrm{r} \mathrm{m}$. waves were the first, evolving afterwards in one direction into the range of long wave-lengths up to $\mathrm{I} \cdot 5 \mathrm{~m}$. waves, and in the other direction in to the range of short wave-lengths- $50 \mathrm{~cm}$., $20 \mathrm{~cm}$., $10 \mathrm{~cm}$. and $3.1 \mathrm{~cm}$. If the exciting agent excites regions of optical thickness equalling $0 \cdot 15$, then the velocity of its motion equals $100 \mathrm{~km}$. $/ \mathrm{sec}$. (in the Press).

B. M. Chikhatchev investigated in 1949 the problem of radio emission of sunspots for $\mathrm{I} \cdot 5 \mathrm{~m}$. and $2 \mathrm{~m}$. waves by means of an interference method. It was established that the radioemitting regions are located above the groups of sunspots at an altitude of 0.4 solar radii over the photosphere. The intensity of the magnetic fields of the radio-emitting spots exceeds 2000 gauss (in preparation).

A. E. Salomonovitch and T. A. Shmaonov investigated the low-frequency region of the spectrum of the internal noise of a modulation radiometer. A conclusion reached was that the frequencies of modulation must not be selected under 50 hertz (in preparation). 
C. E. Khaikin, N. L. Kajdanovsky and M. T. Turusbekov. The position of the "centre of gravity' of radio emission of the Moon for different phases was determined at $3.2 \mathrm{~cm}$. The 'centre of gravity' of radio emission of the Moon coincides for every phase with the geometric centre of the Moon, which shows that the amplitude of the change of the 'radio temperature' of the Moon does not exceed $8^{\circ}$ at $3 \cdot 2 \mathrm{~cm}$.

I. S. Shklovsky (A.J. U.S.S.R. 29, I44, r952) developed a theory of monochromatic radio emission of the Galaxy on $2 \mathrm{I} \mathrm{cm}$., taking into account galactic rotation. In the direction to the centre one may expect an extremely faint absorption line of deuterium at $327 \cdot 4$ Mc. /sec.

I. S. Shklovsky (A.J. U.S.S.R. 30, 15, 1953) showed that the radio emission of the Galaxy consists of two components with different spectra and different spatial distribution: (a) gaseous, forming a flat sub-system, $(b)$ emission from relativistic electrons in interstellar magnetic fields forming a 'spherical' sub-system. The radio emission of the Crab nebula is not thermal. The sources of Cassiopeia-A and Fornax-A are identical with the supernova $369 \mathrm{AD}$ and NGC 1316, respectively. The expected flux of thermal radio emission from the brightest diffuse nebulae are calculated. The absolute and relative powers of various galaxies are also calculated. The latter vary over an extremely wide range.

I. S. Shklovsky (C.R. Acad. Sci. U.R.S.S. 90, 983, 1953) showed the inconsistency of the existing interpretations of the continuous optical emission of the Crab nebula. It is shown that this emission cannot be of a thermal character. It is a continuation of the radio spectrum of this nebula. It is caused by a comparatively small number of relativistic electrons with energies of $\mathrm{IO}^{11}-\mathrm{IO}^{12} \mathrm{eV}$. It follows that the masses of the envelopes of the supernovae do not exceed $0 \cdot I \mathrm{M}$.

I. S. Shklovsky (C.R. Acad. Sci. U.R.S.S. 91, 475, I953; A.J. U.S.S.R. 30, 577, 1953). It was demonstrated that during the outbursts of supernovae enough relativistic particles are formed to provide a sufficient amount of primary cosmic rays in the Galaxy. This is taken as a basis for the development of a new theory of the origin of primary cosmic rays. It is suggested that the envelopes of novae must be sources of radio emission.

I. S. Shklovsky (C.R. Acad. Sci. U.R.S.S. 92, 25, I953). The frequencies of the radio lines $\mathrm{OH}, \mathrm{CH}$ and others, originating in transitions between the components of $\Lambda$-doubiing of the ground rotation levels are calculated. It is shown that these lines should be observed in the radio spectrum of the Galaxy.

I. S. Shklovsky (C.R. Acad. Sci. U.R.S.S. 94, 4I 7, 1954). The sources of class I connected with the galactic supernovae $I 85,369,827$ and 1006 are identified. It is shown that outbursts of supernovae occur in the Galaxy at least once every twenty to thirty years.

I. S. Shklovsky (C.R. Acad. Sci. U.R.S.S. 97, 53, 1954). The source of radio emission connected with the nebula IC 443 , discovered recently, is identified with the Nova 837 .

I. S. Shklovsky 'Photometric paradox for radio emission of the Metagalaxy' (A.J. U.S.S.R. 30, 495, 1953). A relation between radio astronomy and cosmology is established. It is demonstrated that the red shift influences the radio emission of the Metagalaxy.

I. S. Shklovsky (A.C. U.S.S.R. no. 148,3 , I954). The source of radio emission $17-2 \mathrm{~A}$ of the catalogue of Mills $(\lambda=3 \mathrm{~m}$.) is identified with the diffuse nebula NGC 6523 .

I. S. Shklovsky (A.J. U.S.S.R. 31, no. 6, I954) identified source no. 22 of the catalogue of Hanbury Brown with the diffuse nebula IC 1396 , the emission of which is thermal. He also found that the nebula NGC 6357 is identified with a comparatively powerful source of radio emission, observed in the waves $\lambda=3 \mathrm{~m}$. and $75 \mathrm{~cm}$. The sources of emission of the first and second nebulae are apparently not of thermal character. These two nebulae are, obviously, remnants of outbursts of supernovae.

I. S. Shklovsky (A.J. U.S.S.R. 3I, no. 6, I954). The hypothesis is advanced and grounds given that the radio emission of clusters of galaxies and super-galaxies is caused by relativistic electrons moving in intergalactic space.

I. S. Shklovsky (C.R. Acad. Sci. U.R.S.S. 98, 353, I954). The formation of relativistic electrons can take place in the front of a shock wave. The source Cassiopeia-A is a decelerated envelope of a supernova. A quantitative theory of the origin of radio emission from collisions of galaxies is given for Cygnus-A. 
P. I. Bakulin and I. S. Shklovsky (A.J. U.S.S.R. 32, no. I, 1955, in the Press). An ephemeris for different places in the U.S.S.R. of occultations by the Moon of sources of radio emission, namely of the Crab nebula and IC 443.

I. S. Shklovsky (A.J. U.S.S.R. 31, 483, I954). Discussion with Baade and Minkowski concerning their interpretation of radio astronomical data of observations.

I. S. Shklovsky (A.C. U.S.S.R. in the Press). A proof is given that the majority of metagalactic discrete sources of radio emission cannot be the colliding galaxies of the type of Cygnus-A. They are, obviously, similar to NGC 4486 .

G. A. Shajn and I. S. Shklovsky, 'Identification of the continuous sources of radio emission with $\mathrm{H}$ II regions' (A.J. U.S.S.R. in the Press). A majority of the extended sources observed by Bolton and his collaborators are identified with the extended $\mathrm{H}$ II regions. One source is being identified with the cluster of galaxies in the Coma-Virgo region.

I. S. Shklovsky, 'Interpretation of the radio emission of the galaxy NGC 4486' (C.R. Acad. Sci. U.R.S.S. in the Press). The cause of emission is the relativistic electrons formed in the central jet of that galaxy. The optical luminosity of this jet is caused by the relativistic electrons, which are diffused therefrom. This explains the ellipticity of that galaxy in radio emission.

I. S. Shklovsky, 'Supernovae and radio astronomy'. Publications of the fourth cosmogonical conference, in the Press. A detailed analysis of the problem of radio emission of remnants of supernovae and novae.

S. M. Poloskov (A.J. U.S.S.R. 30, 68, 1953). Questions concerning the possibility of observation of the continuous and monochromatic radio emission of comets were investigated.

V. L. Ginsburg (C.R. Acad. Sci.U.R.S.S. 83, 385, I952). The question of the possibility of explaining the ionospheric excitation by the fall into the ionosphere of interstellar dust and gas is discussed. A method for testing this hypothesis by means of observations of the eclipse of the current of interstellar matter by the Moon is given.

B. N. Gershman and V. L. Ginsburg, 'Mechanism of the origin of irregularities in the ionosphere' (C.R. Acad. Sci. U.R.S.S. in the Press). The possibility that the origin of ionospheric irregularities causing scintillation of radio stars is a result of the formation of a convective instability in the upper part of the F-layer is demonstrated.

G. G. Getmanzev and V. L. Ginsburg (C.R. Acad. Sci. U.R.S.S. 87, 187, 1952). The possibility of explaining the sporadic radio emission connected with sunspots as a result of emission from relativistic electrons moving in the magnetic field of the spots is discussed.

V. L. Ginsburg and M. I. Fradkin (C.R. Acad. Sci. U.R.S.S. 92, 531, 1953). From the data concerning the intensity over the spectrum of the general galactic radio emission the energy spectrum of the electron component of the cosmic rays in the Galaxy was obtained. A number of other questions connected with the electron component of cosmic rays are discussed in the article. It is shown that the cut-off of the spectrum of cosmic rays observed on the Earth in high latitudes can be connected with the presence of the magnetic moment of the solar system, and not of the Sun.

V. L. Ginsburg (C.R. Acad. Sci. U.R.S.S. 92, 727, r953). The high efficiency of the statistical mechanism of acceleration of particles in the expanding envelopes of novae and supernovae is pointed out. This mechanism leads, obviously, to the formation in the envelopes of the supernovae of a large number of relativistic electrons, responsible for the intense radio emission of these envelopes.

V. L. Ginsburg (C.R. Acad. Sci. U.R.S.S. 92, I x33, I953). A hypothesis connecting radio emission from the remnants of supernovae with the magnetic Bremsstrahlung of relativistic electrons is discussed. The possibility of discovering radio emission of novae is mentioned, as also the connexion of the cosmic radio-emission problem with the theory of origin of cosmic rays. The importance of measuring the polarization of the radio emission of the remnants of supernovae is stressed.

V. L. Ginsburg, 'The origin of cosmic rays and radio astronomy' (Progress of Physical Sciences, 51, 343, 1953). A general survey and a critical analysis of the modern state of the theory of origin of cosmic rays is given. Attention is paid, chiefly, to the stage of that problem connected with the progress of radio astronomy. A picture of the origin and evolution of 
cosmic rays in the Galaxy is given in conclusion and the problems for further experimental and theoretical studies in this domain outlined.

V. S. Troitzky, 'Concerning the theory of radio emission of the Moon' (in the Press). A relation between the distribution of the intensity of radio emission and the integral radio emission of the Moon, depending on its phase, is deduced. The polarization of the total radio emission is calculated.

V. S. Troitzky, G. G. Getmanzev, V. T. Bobrick, M. R. Zelinskajam and V. L. Rakhlin (prepared for Press). Experimental curves of radio observations during the eclipses of 25 February 1952 and 30 June 1954 on $3.2 \mathrm{~cm}$., $10 \mathrm{~cm}$., and $1 \cdot 5 \mathrm{~m}$. are given. Results of calculations of the intensity of radio emission are also communicated.

V. S. Troitzky, V. L. Rakhlin, V. T. Bobrick and A. M. Starodubtzev, 'Radio astronomical station of Gorky' (prepared for Press). A description of the radio telescopes for $1 \cdot 5 \mathrm{~m}$., ro $\mathrm{cm}$., and $3.2 \mathrm{~cm}$. is given. Results of the absolute measurements of the intensity of radio emission of the Sun are also given.

V. S. Troitzky, 'The theory of zero-method measurements of faint noise' Journal of Technical Physics (in the Press). The condition of balance of zero measuring devices, applied in radio astronomy for various characteristics of the compared intensities, is given.

M. P. Zelinskaja, 'Determination of the properties of surface rocks of the Moon according to their radio emission in the $3.2 \mathrm{~cm}$. wave-lengths' (in the Press). No relation between the emission and the phases of the Moon with a precision of $\pm 7 \%$ was observed. The mean effective temperature of the Moon equals $140^{\circ}$. The surface layers are of a dispersed structure with thermal conductivity less than $\mathrm{I} \cdot 4 \times \mathrm{IO}^{-4} \mathrm{cal}$. per $\mathrm{cm}$. sec. degree.

V. S. Troitzky, 'Noise in a charged line' (in the Press). Errors of temperature measurement caused by the internal noise of the input resistance are investigated by means of radio astronomical instruments.

G. G. Getmanzev and K. S. Stankevitch, 'Monochromatic radio emission of the centre of the Galaxy on $9 \mathrm{r} \cdot 6 \mathrm{~cm}$.' (prepared for Press). Data concerning the intensity of monochromatic radio emission of deuterium are given.

G. G. Getmanzev, 'On the division of the observed cosmic radio emission into the galactic and metagalactic components' (in the Press).

G. G. Getmanzev (C.R. Acad.Sci. U.R.S.S. 83, 557, 1952). It was shown that the emission of cosmic electrons in the interstellar magnetic field explains the main properties of the observed cosmic radio emission.

G. G. Getmanzev, 'On the question of "electrono-magnetic" mechanisms of cosmic radio emission' (in the Press). The degree of polarization of radio emission and the distribution of the sources of cosmic rays are calculated, assuming that radio emission is connected with cosmic electrons.

G. G. Getmanzev, 'Concerning the spatial distribution of the sources of primary cosmic rays' (in the 'Press). On the assumption of the 'electrono-magnetic' nature of cosmic radio emission the spatial distribution of the sources of primary cosmic rays is established.

G. G. Getmanzev and V. L. Ginsburg (C.R. Acad. Sci. U.R.S.S. 87, 187, 1952). It was shown that the 'electrono-magnetic' mechanism of radio emission can be realized for the conditions that exist in a sunspot.

G. G. Getmanzev, 'Cosmic electrons as a source of radio emission of the Galaxy' (in the Press).

V. V. Zhelezniakov, 'On the question of sporadic radio emission of sunspots' (in the Press). Polarization and spectrum of radio emission were found on the assumption that sporadic radio emission is caused by the emission of relativistic electrons. 


\section{Report of the meetings. 30 August and 3 September 1955}

President: Dr J. L. Pawsey.

SeCretary: J. P. Wild.

The Commission held two meetings which were devoted to matters requiring cooperative arrangements. Between the meetings items were considered in detail by sub-committees appointed at the first meeting. Recent work had been reported at the three-day Symposium on Radio Astronomy held during the previous week at Jodrell Bank under the sponsorship of the Commission.

The following items were discussed:

(I) Draft Reports. Since the compilation of the Draft Report the President had received a special report on Radio Astronomy being carried out in the U.S.S.R. The Commission agreed that since many of its members were unfamiliar with this work, the report of the U.S.S.R. should be printed in the Transactions as an appendix to the President's report. With this addition the report was approved.

(2) Terminology. The Commission recommended that the I.A.U. should adopt the terminology for Radio Astronomy recommended by U.R.S.I. (pp. 576-7).

(3) Galactic Co-ordinates. It was pointed out by Westerhout that recent galactic radio observations, particularly those in the $2 \mathrm{I} \mathrm{cm}$. line of hydrogen, had emphasized the need for a new system of galactic co-ordinates having the Equator lying as close as practicable to the galactic plane and the zero meridian passing through the galactic centre. The Commission appreciated that within the next few years the true positions of the galactic pole and centre would probably be known with considerably greater accuracy than at present. It recommended, jointly with Commission 33, that the Executive Committee appoint a Sub-commission to investigate the desirability of such a revision, nominating Dr Blaauw, Dr Pawsey and Dr Westerhout as members of the Sub-commission.

In the meantime, arrangements were suggested by Westerhout for compiling standard tables (I) converting celestial to galactic co-ordinates (present system) and (2) giving corrections for the Earth's motion. Proposals for compiling such tables were considered by a sub-committee including: Westerhout, Hagen, MacRae, Reiz, Pawsey, Bok, Heeschan, Helfer, Lilley. The Commission requested Westerhout to co-ordinate the prompt preparation of these tables.

(4) Frequency Allocations. The Commission vigorously endorsed the efforts of U.R.S.I. to obtain such clear frequency bands as are reasonably necessary for radio astronomical research. It recommended that the Executive Committee request U.R.S.I. to renew its negotiations with C.C.I.R. and at the same time emphasize to C.C.I.R. the importance which it attaches to decisive action by I.T.U. to obtain the necessary clear frequency bands. A report was prepared by an appointed sub-committee informing U.R.S.I. and national authorities of the Commission's views in more detail (see Appendix I).

(5) $A$ Symposium for I958. The Commission favoured a proposal that a Symposium on Radio Astronomy should be held immediately prior to the tenth General Assembly of the I.A.U. in I958. In a recommendation to the Executive Committee, it suggested that such a Symposium lasting one week be held in France and sponsored jointly by the I.A.U. and U.R.S.I.

(6) Bibliography and Publications. The Commission agreed that the Bibliography on Radio Astronomy prepared at Cornell University (Editor, Martha Stahr Carpenter) has been very helpful to workers in the field and approved its continuance. It was agreed that its present form and content are satisfactory. Since this form of publication necessarily involves a time-lag of one to two years, it was further recommended that an up-to-date list of references without abstracts be issued quarterly with as small a delay as possible. It was considered desirable that where possible such a list should include the titles of papers accepted by journals before their publication. In order to ensure the smooth working of these bibliographies, the Commission appealed to all authors to send 
promptly the following information direct to the Editor, Martha Stahr Carpenter, Department of Astronomy, Rockefeller Hall, Cornell University, Ithaca, N.Y., U.S.A.:

(a) Prior to publication, titles of papers accepted for publication by journals and the name of the journal.

(b) As soon as possible after publication reprints of each paper together with an abstract in English.

The Commission discussed a suggestion by Bok that requirements existed for one or more journals devoted specifically to radio astronomy. Two points of view were expressed: (I) that such a journal could usefully exist side by side with astronomical journals, and (2) that the separation of radio astronomical material from astronomical material was questionable.

The Commission decided to take no action at present.

(7) Standardization of Intensity Measurements. Considering the serious discrepancies between the absolute values of the flux density of discrete and other sources of radio emission obtained by different observers, the Commission attached considerable importance to the problem of making accurate intensity measurements. A sub-committee was appointed to review the problem and suggest means of approaching it. The report (Appendix 2) was adopted by the Commission.

(8) Experiments not requiring large aerials. The Commission discussed a suggestion by Ryle that a list of useful experiments not requiring the use of large and expensive aerials would be useful for the guidance of workers entering this field of research, but having limited experience and funds. The Commission appointed a sub-committee to suggest a list of such experiments. The sub-committee's report (Appendix 3) was adopted by the Commission.

(9) Computation of lunar occultations. It was brought to the notice of the Commission by Ryle that the British Nautical Almanac Office has recently started a programme for the computation of the circumstances of the lunar occultations of radio stars, and that the positions of a number of radio stars from the Cambridge Survey which lie near the ecliptic have been given to this Office. The addresses of a number of observatories which were thought to be interested have already been given to the N.A.O. If other observatories are interested, they should get in touch with the N.A.O. giving the location and heights of their apparatus. Seeger commented that during the next year or so there will be a series of lunar occultations of the Crab nebula and suggested that these should be noted by observers. These occultations had been discussed by Link in the literature.

(Io) Other Business. The Commission requested the President to include in his next Draft Report a complete world-wide list of radio observatories.

The President was requested to circulate mimeographed copies of the minutes of these meetings to all members of Commission 40 and other interested persons as soon as possible after the meeting.

\section{APPENDIX I}

\section{FREQUENCY ALLOCATIONS}

The allocation of frequency bands for radio astronomy has been the subject of negotiations between U.R.S.I. and C.C.I.R. since I948. The rapid progress made in recent years has led to the recommendation by the I.A.U. that immediate action be taken to provide protection for future radio astronomical work. Discussions in Commission 40 of the I.A.U. have led to the following conclusions.

(I) The difficulties of providing internationally agreed bands is well understood, and it is felt that the problem can only be approached in terms of a general directive from the I.T.U. to its members.

(II) It is suggested that national authorities should base their plans on the following general requirements of radio astronomy. 
(I) Clearance is in general only necessary in the neighbourhood of the radio astronomical observatories; the radius cannot be defined precisely since it depends on the power and frequency of the transmitter and the nature of the terrain.

(2) Approximately one frequency band per octave should be provided up to $30,000 \mathrm{Mc}$. $/ \mathrm{s}$. Owing to the possibility of interference from the harmonics of other services, it is desirable that the frequencies should be harmonically related.

(3) The bands should include the two known line frequencies of $1420 \cdot 4$ and $327 \cdot 4 \mathrm{Mc} / \mathrm{s}$. The possibility of observing other lines at higher frequencies has been predicted theoretically, but it is felt undesirable to make general recommendations concerning the clearance of these bands until they have been observed.

(4) The band-widths should be of the order of $r \%$ of the centre frequency. In the case of the $\mathrm{I}_{420} 4 \mathrm{Mc}$. $/ \mathrm{s}$. line, it is desirable that the band be extended to $\mathrm{I}_{4} 00 \mathrm{Mc}$. $/ \mathrm{s}$.

(5) For frequencies less than $40 \mathrm{Mc}$. $/ \mathrm{s}$. the problem can no longer be settled on a national basis, and internationally agreed bands are necessary.

It is understood that a clear band in the neighbourhood of $18 \mathrm{Mc} / \mathrm{s}$. is required for a warning service for the I.G.Y. programme on solar flares. The details will be provided by the I.G.Y. on solar and terrestrial relationships this month in Brussels.

The permanent provision of this band would satisfy the demands of radio astronomy in this octave.

(6) The provision of a frequency below ro Mc./s. presents especial difficulties. It has been suggested that the guard band of the 5 and ro Mc./s. Standard Frequency Transmissions might be used for this purpose; it is understood, however, that interfering signals often appear in these guard bands, and action would be necessary to keep them clear.

$\begin{array}{cl}\text { Signed: LAFfineur } & \text { RyLE } \\ \text { HageN } & \text { Vitkevitch } \\ \text { DE Voogt } & \end{array}$

APPENDIX 2

\section{NOTES ON STANDARDIZATION OF INTENSITY MEASUREMENTS}

Observations of both the integrated radiation and the discrete radio sources have now been made over a wide range of frequencies. A comparison of the results obtained shows that there are serious discrepancies between the flux densities obtained by different observers. A knowledge of the spectra of the sources is of great importance in connexion with theories of the radio emission and it is now important to examine means for improving the accuracy of such observations.

In section I below, the basic methods of measurement are discussed, and a system is proposed for the intercomparison of results obtained by different observers; the method suggested may also be of value to those who wish to standardize their apparatus without developing the techniques discussed in section II.

I. The comparison of standards. The measurement of the surface brightness of the integrated radiation depends simply on the establishment of a standard of noise power; the measurement of the flux density from a discrete source requires in addition a knowledge of the aerial gain. Methods for comparing both quantities are discussed below; the absolute calibration will be discussed in section II.

(a) Noise power. The simplest method of relating standards of noise power is based on a measurement of the difference of surface brightness in two areas of the sky. It is necessary that the areas selected should be effectively uniform over an area larger than the reception pattern of the aerial used; for measurement at lower frequencies it would be necessary to avoid areas at galactic latitudes less than $20^{\circ}$, but at the higher frequencies $(>400 \mathrm{Mc} . / \mathrm{s}$.) the small intensity would probably make it necessary to use additional areas near the galactic plane

Measurements of the continuous radiation in the neighbourhood of the $1420 \mathrm{Mc}$. /s. hydrogen line might also be used to standardize a noise source which by a switching method could be 
used to calibrate the line intensities. Alternatively, direct integration of the line contours obtained with standardized high resolution systems, to allow both for band-width and beam-width may prove more practicable.

(b) Aerial Gain. Measurements of the aerial temperature produced by a discrete source of known flux density enable the derivation of the aerial gain. In practice difficulties may arise due to: (i) the difficulty of distinguishing between the contribution of the source and irregularities in the integrated radiation, (ii) the finite angular extent of the source, (iii) the effect of nearby weak radio sources.

Interferometric methods may be used to reduce (i), but this may in turn involve (ii).

Observations with single aerials of low resolving power are probably only reliable in the case of the intense source in Cassiopeia $\left(23 \mathrm{~N}_{5} \mathrm{~A}\right.$ ), and perbaps M.87 (I2NIA). The use of interferometers having apertures of the order of $30 \lambda$ would allow good observations of the Cygnus source $\left(\mathrm{r}_{9} \mathrm{~N}_{4} \mathrm{~A}\right)$, the Crab Nebula $\left({ }_{0} \mathrm{~N}_{2} \mathrm{~A}\right)$ and that in Centaurus $\left(\mathrm{I}_{3} \mathrm{~S}_{4} \mathrm{~A}\right)$. Other sources would only be valuable for somewhat larger instruments, owing to limitation (iii). Observations with larger interferometric spacings may involve (ii), and it will be necessary to consider the decrease of amplitude with resolving power; data on this are still incomplete.

At centimetric wave-lengths, where pencil beam systems of high resolution are normally used, all the sources considered should prove satisfactory, as well as the Orion nebula (o5SoA) and the Moon.

II. Absolute standards. It is clear from a comparison of the present observations that the present standards of both noise power and flux density are unsatisfactory. Every effort should be made to improve the accuracy of absolute measurements at all frequencies. Standards of noise power based on temperature-limited diodes have proved inaccurate, and it is concluded that a thermal source provides the only reliable standard of noise power. In the derivation of the surface brightness of the background radiation it is also necessary to establish the zero level, and this required accurate measurements of the losses and impedance matching of the aerial and thermal load.

The standardization of flux density requires the calculation or measurement of the gain of the aerial: so far the most reliable results have been obtained from calculations of simple aerials (at the low frequencies) and of horns at the higher frequencies.

In parallel with programmes of this type it is important that a study be made of the most suitable areas for intercomparison of the integrated radiation; this investigation should determine the accuracy with which the measured aerial temperature may vary for aerials of different resolving power, and should include both the effects of non-uniformity in the contours, and the presence of discrete sources.

Whilst it is impossible to make any final suggestions at the present time, it is suggested that the following standard sources and areas might be examined; from the results obtained it may then be possible to present at the next General Assembly of the I.A.U. a recommended list.

(a) Standard areas

(i) Low frequencies: $l=190^{\circ} b=+37^{\circ}$ low temperature,

$$
l=320^{\circ} b=+35^{\circ} \text { high temperature. }
$$

These areas have the advantage that they are at approximately the same declination near the equator and are therefore accessible to both hemispheres.

(ii) High frequencies: Plot a profile through the galactic plane near R.A. $18^{h}$, Dec. $-12^{\circ}$; aerials of lower resolution may then be calibrated by integration of surveys obtained with high resolving power.

(b) Standard sources
(i) $23 \mathrm{~N}_{5} \mathrm{~A}$
(ii) I2NIA
(iii) $\mathrm{I}_{9} \mathrm{~N}_{4} \mathrm{~A}$
(iv) $05 \mathrm{~N} 2 \mathrm{~A}$

(v) ${ }_{3} \mathrm{~S}_{4} \mathrm{~A}$

(vi) Orion nebula
(vii) Moon

\begin{tabular}{|c|c|}
\hline Signed: RyLE & Bolton \\
\hline $\begin{array}{l}\text { SEEGER } \\
\text { HADDOCK }\end{array}$ & BuRKE \\
\hline
\end{tabular}




\section{APPENDIX 3}

POSSIBLE OBSERVATIONS NOT REQUIRING LARGE AERIALS

There are a number of important investigations in radio astronomy which do not require the use of large instruments. It has been suggested that it might be valuable to indicate a number of such investigations for the consideration of those smaller observatories which are now planning radio astronomical programmes.

Some of the observations listed below, under $(a)$, relate to variable phenomena and these require that observations be made at a large number of different places in order to obtain a more complete picture than can at present be obtained; some of the observations in this category might also be suitable for consideration by amateur organizations.

Others, listed under $(b)$, represent investigations in which high standards of accuracy or technique may be involved; they may lead to difficult and extended programmes yet do not require the use of large aerial systems.

(a) (i) Observation of the radio emission from Jupiter; first to provide an effectively continuous watch on activity, so that the true time variation can be determined; and secondly to extend the observations to both higher and lower frequencies to determine both the average spectrum and also the correlation between the fluctuations at different frequencies.

(ii) Observation of the scintillation of radio stars to provide a wider picture of the dependence on time and geographical position. Whilst largely of geophysical interest, it is of direct importance astronomically as a limitation to low-frequency observations, whilst the origin of the irregularities is still quite unknown.

(iii) Measurements of ionospheric absorption by observing galactic radiation; this is of value as a warning of solar activity in addition to its geophysical importance.

(iv) There are at present no regular observations of the Sun at frequencies below 200 Mc./s. except in England; relatively simple equipment has been shown to be successful at frequencies as low as $40 \mathrm{Mc}$./s. and further observations at other longitudes would be of great value.

(v) Regular observations using interferometers to determine the position, angular size and polarization of the sources associated with sunspots should be established over a wide range of frequencies.

(b) (i) Absolute measurements of the flux density of both discrete sources and the background cosmic radiation are of great importance, especially in the range $30-300 \mathrm{Mc}$. $/ \mathrm{s}$.

(ii) The observation of the dynamic spectra of solar bursts which have been made in Australia, represent an important method of solar research; it is important to develop similar methods for the higher frequencies.

(iii) It would be valuable if observations could be made to confirm the suspected variations in the flux density of the radio source in Hydra and that reported by Kraus.

Signed: RYLE

FLEISCHER

BolToN 